\title{
Research on Wide Band Smoke Materials
}

\author{
Yuting Liu, a , Qian Zhang ${ }^{1}$ and Zhining Zhao ${ }^{2}$ \\ ${ }^{1}$ Shijiazhuang Mechanical engineering College, Shijiazhuang, 050003, China \\ 2Joint staff college, National Defence University, Beijing, 100856, China \\ a541014105@qq.com
}

\begin{abstract}
Keywords: Passive jamming, Wide band, Smoke material.
\end{abstract}
\begin{abstract}
In the modern war electromagnetic spectrums are used by many kinds of guided weapons involving radio, microwave, infrared, laser, etc. To countermine such weapon systems, the smoke jamming technology is highly paid attention to because of its simple operation, obvious effectiveness and high efficiency cost ratio. This paper mainly sums up the research advances of wide band smoke materials, centers on expatiating the operation mechanism of smoke jamming technology, and analyzes the development trend of smoke jamming technology.
\end{abstract}

\section{Introduction}

From the Gulf War, the Iraq war and other modern local war, people are fully awared of the extraordinary combat effectiveness of precision-guided weapons.Advanced guidance systems and control systems have changed the way of conventional operations. The US military uses radar detection technology, opto-electronic reconnaissance equipment and precision-guided weapons to quickly destroy enemy military command agencies, and other important military targets through stealth penetration, precision strike, and nighttime operations. At present, there are thousands of precision-guided weapons in foreign equipments, including infrared, laser, TV, GPS, radar, millimeter wave, and dual-mode or multi-mode composite guidance.Smoke jamming technology for its obvious effectiveness, high cost ratio is widely used in the field of photo-electric antagonism. With the development and application of multi-mode composite guidance technology, the formation of ordinary smoke screen is difficult to achieve the desired jamming effectiveness. The new spectrum of hyper-spectral is a new challenge to the development of smoke jamming technology ${ }^{[1]}$.In order to meet the needs of future information technology operations, the new smoke materials of wide band need to be developed, and the research of wide band smoke jamming technology need to be actively carried out .

\section{Introduction To Wide Band Smoke Jamming Techonology}

Smoke interference is to form aerosol particles, attenuation through the smoke agents or interference materials in the air to change the transmission characteristics of electromagnetic waves to achieve the interference to guided weapons. Traditional smoke screen, such as yellow phosphorus smoke, red phosphorus smoke, the infrared HC hot smoke agents and metal powder and other cold smoke agents, the millimeter wave jamming materials,etc.Due to the rapid development and popularization of multi-mode composite guidance technology, the traditional smoke screen is difficult to meet the needs of future operations for its simple interference mechanism, low extinction efficiency . In response to the threat of multi-mode compound guided weapons, wide band smoke jamming is often used. Wide band smoke jamming technology refers to the interference by spraying a number of smoke agents or interference materials, to achieve wide band interference. It can be divided into two types,including combination interference and composite interference. Combined interference is to mix the different bands of materials in a certain proportion, the formation of the composite smoke screen finally meet the purpose to interference multi-mode composite guided weapons. However, the compatibility of each material in the interference system should be considered as this method, and the aerodynamic characteristics of different materials is not the same after the 
dispersion,it is easy to be identified by the composite seeker.Composite interference is to use a composite smoke interference material, to achieve wide band interference.It not only achieve effective interference in a broadband, but also ensure the uniformity and stability of interference system, to eliminate the impact of different interference materials.

\section{The Operation Mechanism of Wide Band Smoke Screen}

Infrared radiation in the 1um $\sim 3 \mathrm{um}, 3 \mathrm{um} \sim 5 \mathrm{um}, 8 \mathrm{um} \sim 14 \mathrm{um}$ band with high transmittance, infrared observation equipment and guided weapons generally work in this band range. Laser-guided weapons are mainly work at $1.06 \mathrm{um}$ and $10.6 \mathrm{um}$. The millimeter-wave radar mainly work in the $3 \mathrm{~mm}$ and $8 \mathrm{~mm}$. Wide band smoke screen should have a greater attenuation near the above bands. It mainly interference with the scattering of materials in the visible light band. For the order of microns, it is the result of absorption and scattering together.It is the result of absorption in the millimeter wave band $^{[2]}$.

\subsection{Absorption Attenuation}

The energy of the atom includes the total rotational energy, the vibrational energy of the atoms in the equilibrium position, and the kinetic energy of the electrons in the atom relative to the atom nucleus. When the electromagnetic wave incident smoke particles, if the energy is equal with energy level of Particle atom, atoms will produce the corresponding energy level transition. When the electromagnetic wave interacts with the smoke screen, the electromagnetic energy of the incident electromagnetic wave is converted into other forms of internal energy (such as thermal energy) due to a series of loss mechanisms such as conduction loss, dielectric loss, magnetic loss and resonance loss.For discrete particle, it cludes certain specific groups and chemical bonds the groups and chemical bonds will obtain energy for the electrical vector of the electromagnetic waves, Which causes the resonant atoms or molecules in the medium particle structure to produce forced vibrations. When the atoms or molecules that are forced to vibrate collide with other atoms or molecules, the vibrational energy is converted into translational kinetic energy.

\subsection{Scattering Attenuation}

When the electromagnetic wave incident in the medium, the incident radiation energy part of the media particles will be intercepted to obtain the energy,then it emit in all directions. When the wavelength of the electromagnetic wave is much larger than the particle radius, the resulting scattering is called Rayleigh scattering. When the particle size is equal to or greater than the incident wavelength, the scattering of the particles to the electromagnetic wave can be explained by the Mie theory (Mie).

It can be seen that the smoke agents or materials is the key to solve the wide band interference. The material with high conductivity or the imaginary part of the permeability can produce a large absorption attenuation to the electromagnetic wave.The conductance or absorption of interference particles can be improved through chemical process. The interference material has certain specific groups and chemical bonds, and the particles have the appropriate size, which are more likely to have a better scattering characteristics to achieve wide band interference.

\section{Research Advances of Wide Band Smoke Materials}

\subsection{Combined Smoke Materials}

Varmo, Kjell $\mathrm{O}^{[3]}$.developed a wide band smoke material, which anti-infrared interference materials are the aluminums and scaly brass powders, anti-millimeter wave materials are small dipoles with a length of $1 \mathrm{~mm}$ and $1.5 \mathrm{~mm}$.

Feistel company invented a wide band smoke agent. It consists of basic smoke agent and auxiliary smoke agent, the basic smoke agent formula is as follows:ammonium chloride ( $5 \sim 25 \%$ ), polyvinyl chloride (PVC) $(0 \sim 25 \%)$, metal oxide ( $\mathrm{ZnO}, \mathrm{MgO}$ 5 40\%), natural rubber $(5 \sim 30 \%)$. This agent has the function of attenuating visible and infrared radiation.It alsao has the advantages of non-toxic, simple structure of charge, good distribution of smoke agent. 
Wulvik ${ }^{[4]}$ introduced a method of using a mechanical method to disperse wide band materials. The addition of chaff (aluminum-coated glass fiber, corresponding to the wavelengths of $36 \mathrm{GHz}$ and 94 $\mathrm{GHz}$ ) in the aerosol mixture can shield visible light, infrared and millimeter waves (up to 10 to 15 $\mathrm{mm})$ at the same time.

R.D.Giglia ${ }^{[5]}$ invented radar and infrared composite smoke materials. This materials consists of a metal-combustible activated carbon fiber cloth. The activated carbon fiber cloth is composed of a metallized combustible activated carbon fiber and an incombustible adhesive reinforcing fiber.The carbon fibers of the coated metal have a diameter of 4 to $40 \mathrm{~m}$ and a length of 1 to $30 \mathrm{~mm}$.

\subsection{Composite Smoke Materials}

At present, wide band combined smoke materials mainly include fiber materials, expanded graphite, graphite intercalation compounds conductive polymer, and nano-materials, etc.

NICO company developed a new wide band smoke agent NG19.It can be used to interfere with visible light, infrared, millimeter wave multiple bands. The fuming agent is based on expandable graphite and is acidified with sulfuric acid.

U.S. Patent No. 6,578,492 ${ }^{[6]}$ describes an interfering material that can interfere with visible, infrared, and millimeter waves, and attenuates up to $20 \mathrm{~dB}$ for $35 \mathrm{GHz}, 94 \mathrm{GHz}, 140 \mathrm{GHz}$, and 220 $\mathrm{GHz}$ bands. The conductive fiber is composed of carbon fiber, metal fiber, ceramic fiber, glass fiber . The conductive fiber is prepared by adding the conductive fiber into the traditional smoke agent as a dipole. Fiber length in the $1-30 \mathrm{~mm}$, diameter is $0.001-0.1 \mathrm{~mm}$.

Thompson ${ }^{[7]}$ developed a conductive polymer as wide band smoke material. The interference material of the metal deposited on the surface of the particles was prepared by ultrasonic method, and the interference band is $0.3 \sim 40 \mathrm{GHz}$. The microparticles are predominantly polymeric materials such as polypropylene, polyethylene, polyurethane, nylon, polyacrylamide or copolymers of these polymer monomers. In addition, the microparticles include carbon-based materials such as carbon fibers and graphite. The particle size distribution is from $0.001 \mathrm{um}$ to $1 \mathrm{~mm}$, and the morphology of the particles is varied and fibrous and flaky.

$\mathrm{Wu}{ }^{[8]}$ developed a carbon fiber / graphite, carbon fiber / bamboo charcoal and other composite materials composed of smokes agent.The film was subjected to a thermo-forming process to produce $15 \mathrm{~cm} \times 15 \mathrm{~cm} \times 0.2 \mathrm{~cm}$ film to test the attenuation of millimeter waves $(8 \mathrm{~mm})$ and infrared ( 3 to 5 um and 8 to 12 um).

The relative data ${ }^{[9,10,11]}$ show that some nano-materials have high electrical conductivity, ferromagnetism, and strong absorptivity has great application value in the wide band smoke interference technology. Compared with the combination of interfering materials, composite smoke materials charge structure is simple, wide range of loading, the formation of uniform smoke, the effective duration will also increase.

\section{The Development Trend of Smoke Jamming Technology}

\subsection{Developed New Smoke Materials}

Composite interference material is the key to realize wide band interference.It need to develop new interference materials with a larger attenuation and the appropriate aerodynamic characteristics. On the one hand, it need to strengthen the nano-composite materials, fiber-based conductive polymer, absorbing materials, ultra-light aerosols and other new interference materials research. On the other hand, to seek environmentally friendly agents, non-toxic agents, and easily to break down. Using new chemical preparation process to make the material with appropriate size, density, to ensure that the blank time and reduce the atmospheric environmental conditions such as wind, temperature and other climatic factors .

In the traditional $\mathrm{HC}$ smoke agent by adding some aromatic hydrocarbons and their polymerization or decomposition products, such as polystyrene, phenol naphthalene asphalt and coal tar pitch modified or added cesium and cesium compounds to improve the infrared band shielding ability.Replacement of HC smoke with red phosphorus smoke to eliminate irritation and toxicity of Zinc Chloride Smoke. 
It need to develop copper powder, brass powder, aluminum powder, graphite powder and other conductive powder materials, coated with aluminum, copper and other good conductor of the insulating powder materials and fibrous materials; It need to focus on red phosphorus smoke agent and organic reactants such as halogenated hydrocarbons carbon-rich compounds and hexachlorobenzene.

\subsection{Optimize The Charge Structure}

The traditional smoke agent is generally a single charge, and thus the ignition of the drug, the explosive range of the projectile are relatively simple. In order to meet the requirements of the future compound pharmaceutical dosage, it is necessary to design different charge structure and ignition, transmission fire, blasting element, prepare the corresponding explosion-proof and ignition drug, and make reasonable combination smoke agent evenly spread, such as the formation of multi-spectral smoke screen, but also to launch shielded fires and other chaff clouds.

\subsection{Develop Reasonable Test Methods And Evaluation Indicators}

The evaluation of smoke interference is to test whether it can achieve the intended interference effect, mainly through indoor and field tests. The former mainly to interfere with the material testing, such as fourier spectrometer test, infrared radiometer test, infrared thermal imager test, smoke box test. The field test is to evaluate the final interference effect of the interfering smoke. The interference effect of wide band smoke screen to make an accurate assessment, It need to improve the wide band smoke interference test methods and assessment techniques.

\section{References}

[1]. Changgen Feng, Xiaojing Qiao, Wangchang Li.Research Progress in Smoke Bombs [J]. Science \& Technology Review, Vol.32 (2014), p. 110-115.

[2]. Hui Ren, Xiaojing Qiao, Qingjie Jiao. Study on Interference Materials of Wide Spectrum Smoke[J].Oranance Material Science and Engineering,Vol.6 (2001), p. 61-63.

[3]. Varmo, Kjell O. U.S. Patent No. 5337671 (1994)

[4]. [4]. Wulvik, Erik.U.S. Patent No.5233927 (1993)

[5]. R.D.Giglia.U.S. Patent No.5092244 ( 1992)

[6]. Ernst-Christian Koch, Kaiserslautern (DE).U.S. Patent No.6578492 (2003)

[7]. Thompson G. A, Marx.U.S. Patent No.6726964 (2004)

[8]. Wu Guohui, Chen Baohui, Gong Yanzhang, et al. TW.Patent No.200906932 (2009)

[9]. Diederichs HJ, Brunkow B.WO. Patent No.2011060767 (2011)

[10]. Pengyu Bi ,Yi Wu,Pengyu Nie.Study on Infrared Disturbance Performance of Layered Supramolecule Smoke Material [J].Initiators\&Pyrotechnics, Vol.2 (2015), p. 1-5.

[11]. Chenguang Zhu,Chungen XU,Rui Xue.Study on spatial distribution of burning particles in a Pyrotechnic flame based on particle velocity[J].Journal of Energetic Materials, Vol.4 (2014), p. 252-263. 